\title{
Time course of symptomatic orthostatic hypotension and urinary incontinence in patients with postmortem confirmed parkinsonian syndromes: a clinicopathological study
}

Gregor K Wenning, Christoph Scherfler, Roberta Granata, Sylvia Bösch, Marc Verny, K Ray Chaudhuri, Kurt Jellinger, Werner Poewe, Irene Litvan

Department of Neurology, University Hospital, Innsbruck, Austria

GK Wenning

R Granata

C Scherfler

S Bösch

W Poewe

Raymond Escourolle Neuropathology Laboratory, INSERM U 360, Hôpital de la Salpétrière, Paris, France

M Verny

Department of Neurology, Institute of Psychiatry, London, UK

K R Chaudhuri

Ludwig Boltzmann Institute of Clinical Neurobiology, Vienna, Austria

$\mathrm{K}$ Jellinger

Medical Neurology Branch, National Institute of

Neurological Disorders and Stroke, National Institute of Health, Bethesda, Maryland, USA

I Litvan

Correspondence to: Dr Irene Litvan,

Neuropharmacology Unit, Defense and Veteran Head Injury Program, Henry M Jackson Foundation, Federal Building, Room 714,

Bethesda, MD 20892-9130, USA.

Received 26 October 1998 and in revised form 1 February 1999

Accepted 10 February 1999

\begin{abstract}
Objective-Although both orthostatic hypotension and urinary incontinence have been reported in a number of parkinsonian syndromes, such as Parkinson's disease (PD), multiple system atrophy (MSA), dementia with Lewy bodies (DLB), corticobasal degeneration (CBD), and progressive supranuclear palsy (PSP), differences in the evolution of these features have not been studied systematically in pathologically confirmed cases.

Methods-77 cases with pathologically confirmed parkinsonian syndromes (PD, $\mathrm{n}=11$; MSA, $n=15 ; \mathrm{DLB}, \mathrm{n}=14 ; \mathrm{CBD}, \mathrm{n}=13$; PSP, $n=24$ ), collected up to 1994 , formed the basis for a multicentre clinicopathological study organised by the NINDS to improve the differential diagnosis of parkinsonian disorders. The present study determined the time course-that is, latency to onset and duration from onset to death, of symptomatic orthostatic hypotension, and urinary incontinence in the NINDS series. Furthermore, the diagnostic validity of a predefined latency to onset within 1 year of disease onset of symptomatic orthostatic hypotension or urinary incontinence was analysed.
\end{abstract}

Results-Significant group differences for latency, but not duration, of symptomatic orthostatic hypotension and urinary incontinence were found. Latencies to onset of either feature were short in patients with MSA, intermediate in patients with DLB, CBD, and PSP, and long in those with PD. Symptomatic orthostatic hypotension occurring within the first year after disease onset predicted MSA in $75 \%$ of cases; early urinary incontinence was less predictive for MSA (56\%).

Conclusion-Latency to onset, but not duration, of symptomatic orthostatic hypotension or urinary incontinence differentiates PD from other parkinsonian syndromes, particularly MSA.

(F Neurol Neurosurg Psychiatry 1999;67:620-623)

Keywords: orthostatic hypotension; urinary incontinence; parkinsonism

Symptomatic orthostatic hypotension often occurs in Parkinson's disease (PD) ${ }^{12}$ and multiple system atrophy (MSA), ${ }^{34}$ a common cause of atypical parkinsonism. ${ }^{5}$ It may also be present in dementia with Lewy bodies (DLB). ${ }^{6}$ Other parkinsonian syndromes including progressive supranuclear palsy (PSP) ${ }^{78}$ and corticobasal degeneration $(\mathrm{CBD})^{9}$ are less commonly associated with symptomatic orthostatic hypotension. Its presence alone in a patient with parkinsonism, therefore, seems to contribute little towards a differential diagnosis. The temporal evolution of symptomatic orthostatic hypotension has never been explored systematically in parkinsonian disorders. If present, such time course differences may improve the diagnostic accuracy of clinicians facing parkinsonian patients. Indeed, a recent clinicopathological study from the United Kingdom Parkinson's Disease Society Brain Bank reported early severe orthostatic hypotension in patients with confirmed MSA than in patients with PD; however, latencies and durations of both features were not determined. ${ }^{10}$ In the present study, we retrospectively analysed the temporal evolution of symptomatic orthostatic hypotension in patients with postmortem validated PD, MSA, DLB, PSP, and CBD. Furthermore, we determined whether the early appearance of symptomatic orthostatic hypotension within 1 year of onset of disease improves diagnostic accuracy of these disorders as measured by sensitivity and positive predictive value. Finally, for comparison we analysed the time course of urinary incontinence, another (partly) autonomic feature, in these patients.

\section{Methods}

SAMPLE AND DATA COLLECTION

Seventy seven cases with pathologically confirmed parkinsonian syndromes (PD, $n=11$; MSA, $n=15$; DLB, $n=14$; PSP, $n=24$; CBD, $\mathrm{n}=13$ ) collected up to 1994 , formed the basis for a multicentre clinicopathological study organised by the National Institute of Neurological Disorders (NINDS) to improve differential diagnosis of parkinsonian disorders. Cases with excellent clinicopathological documentation sufficient for the purpose of the present study were selected from the research and clinical neuropathological files of seven medical centres in four countries (Austria, France, United Kingdom, and the United States). The cases met the neuropathological NINDS criteria for the diagnosis of PSP and related disorders ${ }^{11}$ and Kosaka's criteria for Lewy body disease. ${ }^{12}$ Orthostatic hypotension 
Table 1 Demographic characteristics of the sample

\begin{tabular}{lllll}
\hline Disorder $(n=77)$ & No & Sex & Age at onset $(y)^{*}$ & $\begin{array}{c}\text { Disease duration } \\
(\text { months })\end{array}$ \\
\hline PD & 11 & $10 \mathrm{M} / 1 \mathrm{~F}$ & $54.1(11.5)$ & $199(78)$ \\
MSA & 15 & $9 \mathrm{M} / 6 \mathrm{~F}$ & $55.9(10)$ & $74(33)$ \\
DLB & 14 & $9 \mathrm{M} / 5 \mathrm{~F}$ & $66.2(13.8)$ & $90(91)$ \\
CBD & 13 & $6 \mathrm{M} / 7 \mathrm{~F}$ & $63.1(7.8)$ & $92(34)$ \\
PSP & 24 & $15 \mathrm{M} / 9 \mathrm{~F}$ & $63.1(6.9)$ & $79(44)$ \\
\hline
\end{tabular}

${ }^{\star}$ Overall group difference (Kruskal-Wallis): $\mathrm{p}<0.01$; †Overall group difference (Kruskal-Wallis): $\mathrm{p}<0.005$.

Table 2 Median latencies (months) and durations (months) of orthostatic hypotension $(\mathrm{OH})$ and urinary incontinence (UI) in postmortem confirmed parkinsonian syndromes

\begin{tabular}{lclcl}
\hline Disorder & OH: latency & OH: duration & UI: latencyt & UI: duration $\neq$ \\
\hline PD & $166(70-2870) \subseteq$ & $80(7-121)$ & $144(32-186)$ & $37(0-121)$ \\
MSA & $24(0-73)$ & $26(12-76)$ & $12(0-66)^{\star \star}$ & $50(12-98+\dagger$ \\
DLB & $34(26-41)$ & $10(3-16)$ & $38(4-287)$ & $27(16-97)$ \\
CBD & ND & ND & $70(35-84)$ & $39(22-88)$ \\
PSP & $30(0-57)$ & $26(1-82)$ & $54(3-192)$ & $19(3-39) \neq$ \\
\hline
\end{tabular}

Values in parentheses are range.

${ }^{\star}$ Overall difference (Kruskal-Wallis) $\mathrm{p}<0.005$; †Overall difference (Kruskal-Wallis): $\mathrm{p}<0.05$; $\ddagger$ Overall difference (Kruskal-Wallis): $\mathrm{p}=0.09$; (group differences (Mann-Whitney): PD-MSA $\mathrm{p}<0.02$; PD-DLB $\mathrm{p}=0.064 ;$ PD-PSP $\mathrm{p}<0.05$; group difference (Mann-Whitney): PD-MSA $\mathrm{p}<0.005$; PD-DLB $\mathrm{p}=0.086$; PD-PSP $\mathrm{p}=0.061 ;{ }^{\star \star}$ Group difference (Mann-Whitney): MSA PSP $\mathrm{p}<0.05$; MSA - CBD $\mathrm{p}<0.01$; ††Group difference (Mann-Whitney): MSA-PSP $\mathrm{p}<0.02$ \$Group difference (Mann-Whitney): PSP-CBD $\mathrm{p}<0.01$.

Table 3 Validity measures of 1 year latencies for orthostatic hypotension (OH) and urinary incontinence (UI) in postmortem confirmed parkinsonian syndromes

\begin{tabular}{llclccc}
\hline Validity measures & Latencies & PD & MSA & DLB & PSP & \multirow{2}{*}{$C B D$} \\
\hline Sensitivity & OH & 0 & 20 & 0 & 4 & 0 \\
\multirow{2}{*}{ Specificity } & UI & 0 & 33 & 14 & 8 & 0 \\
\multirow{2}{*}{ PPV } & OH & 94 & 98 & 94 & 94 & 94 \\
& UI & 86 & 94 & 89 & 87 & 86 \\
& OH & 0 & 75 & 0 & 25 & 0 \\
& UI & 0 & 56 & 22 & 22 & 0 \\
\hline
\end{tabular}

Data are presented in percentages. PPV=positive predictive value.

was defined as a symptomatic systolic blood pressure drop of $20 \mathrm{~mm} \mathrm{Hg}$ within 3 minutes of standing. ${ }^{13}$ Symptoms of orthostatic hypotension included dizziness, presyncope, and (rarely) syncopal attacks. Blood pressure was recorded at bedside; formal cardiovascular function tests were available in few patients and therefore were not analysed. Urinary incontinence implied partial or complete involuntary bladder emptying in the absence of secondary causes such as outflow obstruction. Because formal urodynamic studies were not always performed, secondary causes may have been present in a minority of patients. Time to onset (months) and duration (months) until death of orthostatic hypotension and urinary incontinence were determined by retrospective chart review (GKW, IL).

STATISTICS

Box plots were applied to determine the distribution of latency and duration data across the groups. Non-parametric statistics (KruskalWallis and Mann Whitney $U$ test) were then used to identifiy significant group differences. Finally, diagnostic accuracy of a predefined latency (urinary incontinence or symptomatic orthostatic hypotension occurring within 1 year from onset of disease) was evaluated by analysing the sensitivity, specificity, and positive predictive value of such predefined latency for the diagnosis of each parkinsonian disorder.

\section{Results}

Table 1 shows the main demographic characteristics of the patients. Medians and ranges of latency and duration of urinary incontinence and orthostatic hypotension are shown in table 2

ORTHOSTATIC HYPOTENSION

Orthostatic hypotension was not recorded in patients with CBD; it rarely occurred in DLB $(15 \%)$, occasionally in PSP, $(45 \%)$ and often in patients with PD $(78 \%)$ and those with MSA $(87 \%)(p<0.0001)$. Latencies were intermediate in patients with MSA, DLB, or PSP compared with long latencies in patients with PD (table 2). Significant overall group differences for latency, but not duration of orthostatic hypotension, were found. Direct group comparisons disclosed significantly longer latencies in patients with PD than in patients with MSA or PSP (table 2).

\section{URINARY INCONTINENCE}

The frequency of urinary incontinence was similar in all patient groups ranging between $62 \%$ and $87 \%$ (PD, $82 \%$; MSA, $87 \%$; DLB, $64 \%$; PSP, $75 \%$; and CBD, 62\%; ( $>>0.05))$. Latencies were short in patients with MSA, intermediate in patients with DLB, PSP, or CBD, and long in patients with PD (table 2). Significant overall group differences for latency, but not duration, of urinary dysfunction were seen. Direct group comparisons disclosed significantly shorter latencies in patients with MSA compared with those with PD, CBD, or PSP (table 2).

EARLY ORTHOSTATIC HYPOTENSION AND URINARY INCONTINENCE

One year latencies for symptomatic orthostatic hypotension achieved poor diagnostic sensitivity for all parkinsonian disorders, and were highest in MSA (20\%) followed by PSP (4\%). Similarly, positive predictive values were higher in patients with MSA (75\%) than (25\%) patients with PSP. Specificity was optimal in MSA (98\%) and suboptimal in PSP (94\%). No patient with PD, CBD, or DLB developed symptomatic orthostatic hypotension within the first year of onset of disease. Diagnostic sensitivity for early urinary incontinence was similarly poor compared with symptomatic orthostatic hypotension, and was highest in MSA (33\%) followed by DLB (14\%) and PSP $(8 \%)$ (table 3$)$. The positive predictive value for early urinary incontinence was slightly less in the patients with MSA and poor or absent in the other patient groups. Specificity was generally suboptimal, in the order of $85 \%$ to $94 \%$.

\section{Discussion}

This clinicopathological study is the first to investigate both frequency and time course of symptomatic orthostatic hypotension and urinary incontinence in a range of postmortem confirmed parkinsonian syndromes including PD, MSA, DLB, CBD, and PSP. Although we recognise the methodological limitations of our study, in particular selection bias toward more severely affected cases, irregular follow up, and 
lack of functional urodynamic and formal autonomic function testing in some patients, the following conclusions seem to emerge from our study: onset latencies of symptomatic orthostatic hypotension and urinary incontinence distinguished PD and atypical parkinsonian syndromes. Latencies were long in patients with PD, extending for more than 10 years. Except for CBD, they were significantly shorter in atypical parkinsonian syndromes, approaching 1-2 years in patients with MSA. Consistent with this finding, a 1 year latency cut off for urinary incontinence and orthostatic hypotension predicted MSA in three of four $(75 \%)$ patients satisfying this criterion. Five of nine patients with MSA with orthostatic symptoms had received levodopa treatment, coadministered with amantadine, bromocriptine, or selegiline in three patients. Although dopaminergic therapy may have triggered or excacerbated orthostatic hypotension in these patients, in the remaining four patients without antiparkinsonian drugs orthostatic symptoms were clearly related to disease. There was one patient (4\%) with PSP with documented symptomatic orthostatic hypotension present from onset of disease and unrelated to drug intake. Generally, the falls in blood pressure seemed milder in PSP than in MSA. However, due to insufficient source data, this could not be analysed systematically. Neither the presence alone nor duration of orthostatic hypotension and urinary incontinence were helpful for differential diagnosis.

Previous studies of neurogenic orthostatic hypotension have largely been devoted to PD and MSA. Bannister and Oppenheimer, in a clinicopathologcial study, first reported that both disorders may account for symptomatic orthostatic hypotension in association with central autonomic failure. ${ }^{1}$ These authors highlighted several clinical features that may be useful for differential diagnosis including young age at onset in patients with PD as well as multiple neurological signs in patients with MSA. Urinary incontinence received little attention in their series, and differences in the time course of orthostatic hypotension were not reported. Neuropathologically, Bannister and Oppenheimer observed marked degeneration of the intermediolateral cell columns of the spinal cord in patients with MSA and those with PD. Lesions were more widespread in MSA affecting the thalamus and hypothalamus as well, and were associated with Lewy body deposition in PD. Almost 20 years later characteristic glial cytoplasmic inclusions were reported by Papp and Lantos in a large number of brains from patients with MSA. ${ }^{14}$

Recent studies confirmed that orthostatic hypotension is usually mild in $\mathrm{PD}^{10}{ }^{16}$; however, a small subgroup of patients seem to have more severe cardiovascular autonomic failure of peripheral origin. ${ }^{17}$ Although the average severity of orthostatic hypotension seems different in large cohorts of PD and MSA, ${ }^{10}$ this information is of limited diagnostic value for the clinician facing a person with parkinsonism and autonomic failure. Non-invasive investigations have been reported to be useful in distin- guishing between MSA and PD in their early stages. The response to clonidine, which is dependent on central stimulation of $\alpha 2-$ adrenergic receptors, is impaired in MSA but preserved in PD. ${ }^{18}$ Food causes a smaller fall in supine blood pressure in PD than MSA. ${ }^{19}$ In general, dopaminergic therapy may exacerbate or trigger symptomatic orthostatic hypotension in parkinsonian patients. Due to the retrospective nature of our study we were unable to consider this issue systematically. Autonomic failure associated with orthostatic hypotension has been recognised as a possible manifestation of DLB ${ }^{20}$ a disorder typically associated with widespread deposition of Lewy bodies also affecting pathways of the autonomic nervous system. Given the considerable clinicopathological overlap of PD and DLB some of the patients with PD and severe autonomic failure reported by Bannister et al ${ }^{1}$ may have had DLB. Severe autonomic dysfunction seems to be uncommon in $\mathrm{PSP}^{7}$ and $\mathrm{CBD}^{21}$; this impression was confirmed by our present study.

Our data demonstrate for the first time highly significant differences in the time course of symptomatic orthostatic hypotension in parkinsonian syndromes. Symptomatic orthostatic hypotension occurring within 1 year of onset of disease predicted MSA in $75 \%$ of patients. In our series, patients with PD or atypical parkinsonian syndromes other than MSA - that is, DLB, PSP, and CBD, rarely or never developed symptomatic orthostatic hypotension during the first year of onset of disease. Therefore, the early development of orthostatic hypotension in patients with parkinsonism strongly suggests MSA.

Bladder dysfunction has again received more attention in PD and MSA than other parkinsonian disorders. In MSA, urinary symptoms result from a combination of detrusor hyperreflexia and urethral sphincter weakness. ${ }^{22}$ Urethral or anal sphincter EMGs often disclose denervation resulting from the degeneration of Onuf's nucleus in the sacral cord. ${ }^{23}{ }^{24}$ In PD, urinary frequency and urgency resulting from detrusor hyperreflexia are common; however, urethral sphincter function is preserved. ${ }^{25}$ These pathophysiological differences probably account for the early appearance of urinary incontinence in MSA compared with PD. Data on urological symptoms in atypical parkinsonian syndromes other than MSA are rare. A Japanese study of nine patients with PSP showed severe abnormalities similar to MSA, including detrusor hyperreflexia, detrusorsphincter dyssynergia, and sphincter denervation. ${ }^{26} \mathrm{~A}$ minority of patients with PSP also develop mild to moderate degrees of denervation affecting the external anal sphincter. ${ }^{27}$

Our comparative study for the first time showed marked time course differences of urinary incontinence between atypical parkinsonian syndromes, particularly MSA and PD. Similar to orthostatic hypotension, early appearance of urinary incontinence within the first year of disease onset suggested MSA although sensitivity and positive predictive values were suboptimal. Sensitivity and positive predictive values for a diagnosis of MSA 
increased at later time points; however, specificity dropped markedly (data not shown).

Taken together, our data show the usefulness of time course differences for symptomatic orthostatic hypotension and urinary incontinence in the early differential diagnosis of parkinsonian syndromes, particularly $\mathrm{PD}$ and MSA. Development of either feature during the first year after disease onset in a patient with parkinsonism is highly suggestive of MSA.

1 Bannister R, Oppenheimer DR. Degenerative diseases of the nervous system associated with autonomic failure. Brain 1972;95:457-74

2 Singer C, Weiner WJ, Sanchez Ramos JR. Autonomic dysfunction in men with Parkinson's disease. Eur Neurol 1992;32:134-40

3 Quinn N. Multiple system atrophy. In: Marsden CD. Fahn S, eds. Movement disorders 3. London: ButterworthS, eds. Movement disorder.

4 Wenning GK, Ben Shlomo Y, Magalhaes N, et al. Clinical features and natural history of multiple system atrophy: an features and natural history of multiple system

5 Quinn NP, Marsden CD. The motor disorder of multiple system atrophy. I Neurol Neurosurg Psychiatry 1993;56: 1239-42

6 McKeith LG, Galasko D, Kosaka K, et al. Consensus guidelines for the clinical and pathologic diagnosis of dementia with Lewy bodies (DLB): report of the consortium on DLB international workshop. Neurology 1996;47:1113-24

7 Litvan I, Mangone CA, McKee A, et al. Natural history of progressive supranuclear palsy (Steele-RichardsonOlszewski syndrome) and clinical predictors of survival: clinicopathological study. $\mathcal{F}$ Neurol Neurosurg Psychiatry 1996;60:615-20

8 Litvan I, Agid Y, Calne D, et al. Clinical research criteria for the diagnosis of progressive supranuclear palsy (SteeleRichardson-Olszewski syndrome): report of the NINDSSPSP international workshop. Neurology 1996;47:1-9.

9 Watts RL, Mirra SS, Richardson EP. Corticobasal ganglionic degeneration. In: Marsden CD, Fahn S, eds. onic degeneration. In: Marsden CD, Fahn S, eds. 1994:282-99

10 Magalhaes M, Wenning GK, Daniel SE, et al. Autonomic dysfunction in pathologically confirmed multiple system atrophy and idiopathic Parkinson's disease: a retrospective comparison. Acta Neurol Scand 1995;91:98-102.

11 Litvan I, Hauw JJ, Bartko JJ, et al. Validity and reliability of the preliminary NINDS neuropathologic criteria for progressive supranuclear palsy and related disorders [see comments]. F Neuropathol Exp Neurol 1996;55:97-105.
12 Kosaka K. Diffuse Lewy body disease in Japan. 7 Neurol 1990;237:197-204.

13 The Consensus Committee of the American Autonomic Society and the American Academy of Neurology. Consensus statement on the definition of orthostatic hypotension, pure autonomic failure, and multiple system atrophy. Neurology 1996;46:1470.

14 Papp MI, Kahn JE, Lantos PL. Glial cytoplasmic inclusions in the CNS of patients with mutilpe system atrophy (striatonigral degeneration, olivopontocerebellar atrophy and Shy Drager syndrome). f Neurol Sci 1989;94:79-100.

15 Papp MI, Lantos PL. Accumulation of tubular structures in oligodendroglial and neuronal cells as the basic alteration
in multiple system atrophy. F Neurol Sci 1992;107:172-82.

16 Sandroni P, Ahlskog JE, Fealey RD, et al. Autonomic involvement in extrapyramidal and cerebellar disorders. Clin Auton Res 1991:1:147-55.

17 Goldstein DS, Holmes C, Cannon RO 3rd, et al. Sympathetic cardioneuropathy in dysautonomias [see comments]. N Engl F Med 1997;336:696-702.

18 Kimber JR, Watson L, Mathias CJ. Distinction of idiopathic Parkinson's disease from multiple-system atrophy by stimulation of growth-hormone release with clonidine. Lancet 1997;349:1877-81.

19 Thomaides T, Bleasdale Barr K, Chaudhuri KR, et al. Cardiovascular and hormonal responses to liquid food challenge in idiopathic Parkinson's disease, multiple system atrophy, and pure autonomic failure. Neurology 1993;43: atrophy, $900-4$.

20 Burkhardt CR, Filley CM, Kleinschmidt DeMasters BK, et al. Diffuse Lewy body disease and progressive dementia. al. Diffuse Lewy body dis
Neurology 1988;38:1520-8

21 Wenning GK, Litvan I, Jankovic J, et al. Natural history and survival of 14 patients with corticobasal degeneration confirmed at postmortem examination. $\mathcal{F}$ Neurol Neurosurg Psychiatry 1998;64:184-9.

22 Beck RO, Betts CD, Fowler CJ. Genitourinary dysfunction in multiple system atrophy: clinical features and treatment in 62 cases. $\mathcal{F}$ Urol 1994;151:1336-41.

23 Pramstaller P, Wenning GK, Smith SJM, et al. Nerve conduction studies, skeletal muscle EMG and sphincter EMG in multiple system atrophy. $f$ Neurol Neurosurg Psychiatry 1995;58:618-21.

24 Palace J, Chandiramani VA, Fowler CJ. Value of sphincter electromyography in the diagnosis of multiple system atroelectromyography in the diagnosis of
phy. Muscle Nerve 1997;20:1396-403.

25 Stocchi F, Carbone A, Inghilleri M, et al. Urodynamic and neurophysiological evaluation in Parkinson's disease and multiple system atrophy. I Neurol Neurosurg Psychiatry
1997;62:507-11.

26 Sakakibara R, Hattori T, Tojo M, et al. Micturitional disturbance in progressive supranuclear palsy. F Auton Nerv Syst 1993;45:101-6.

27 Valldeoriola F, Valls Sole J, Tolosa ES, et al. Striated anal sphincter denervation in patients with progressive supranuclear palsy. Mov Disord 1995;10:550-5. 